\title{
Subsidence monitoring of the Seattle viaduct tunnelling project with Homogeneous Distributed Scatterer InSAR
}

\author{
J Eppler MDA Systems Ltd., Canada \\ M Kubanski MDA Systems Ltd., Canada
}

\begin{abstract}
Subsurface excavations may cause subsidence due to redistribution of stress in the overburden or changes in the groundwater state. In the case of tunnelling under urban areas, this may result in damage to existing high value infrastructure within the surface zone of influence. Synthetic aperture radar interferometry (InSAR) is a useful tool for urban subsidence monitoring because it provides measurements that are wide-scale and ongoing at regular time intervals. However, many existing InSAR methods such as persistent scatterer InSAR and differential InSAR do not provide sufficient spatial resolution to adequately measure short-scale spatial subsidence gradients which represent the greatest risk to infrastructure.
\end{abstract}

MDA Systems Ltd. (MDA) has developed an InSAR method, Homogeneous Distributed Scatterer (HDS-InSAR), which generates higher spatial densities of measured coherent targets compared to many current methods. This is achieved through a novel combination of spatial phase filtering and coherence based target selection. This method exploits both persistent point and coherent distributed scatterers by using adaptive multilooking over statistically homogenous pixel neighbourhoods to improve the spatial resolution of the resulting subsidence maps. Furthermore, a matched parametric temporal model is fit to the deformation signal in order to enhance the detection of small amplitude subsidence. These techniques in combination provide a means for measuring small scale deformations within an urban environment and correlating their occurrence with known deformation drivers.

Results are presented for the ongoing Seattle viaduct tunnelling project which consists of a $3.2 \mathrm{~km}$ long bored road tunnel under the city. Two satellite datasets (RADARSAT-2 Spotlight mode stacks from opposing look directions) are analysed to derive 2D (vertical + east-west) deformation maps over the area of interest spanning a time period from 2012-2015 and these results are correlated with known groundwater extraction events.

\section{Introduction}

This paper presents preliminary results for InSAR-based deformation monitoring of the Washington State Department of Transportation (WSDOT) SR 99 Bored Tunnel Project currently under way in Seattle, USA (WSDOT 2011). The goal of this construction project is to replace an existing above surface elevated road viaduct through downtown Seattle with a $17.5 \mathrm{~m}$ diameter, $3.2 \mathrm{~km}$ long bored tunnel roadway. Figure 1 shows a map of the planned tunnel route.

Boring was initiated in July 2013 and progressed $311 \mathrm{~m}$ by December 2013, at which time the boring machine encountered a mechanical failure which required the construction of a vertical rescue shaft to initiate repairs. Construction of the $37 \mathrm{~m}$ deep by $24 \mathrm{~m}$ diameter shaft involved significant dewatering through wells accessing three aquifer zones: a shallow zone, a mid-level zone and a deep zone (>60 m), extending down to glacial deposits.

The rescue shaft is in an area of Seattle that has experienced historic settlement, as evidenced by tilting streets, roadway cracks and other settlement features. During dewatering for the rescue shaft, additional settlement was noted by project surveyors. If dewatering was a factor in the observed additional 
settlement, the footprint of the dewatering-related deformation could extend beyond the expected boring-related zone of influence and therefore may not be fully covered by the installed ground-based survey networks being monitored by WSDOT and the project prime contractor.

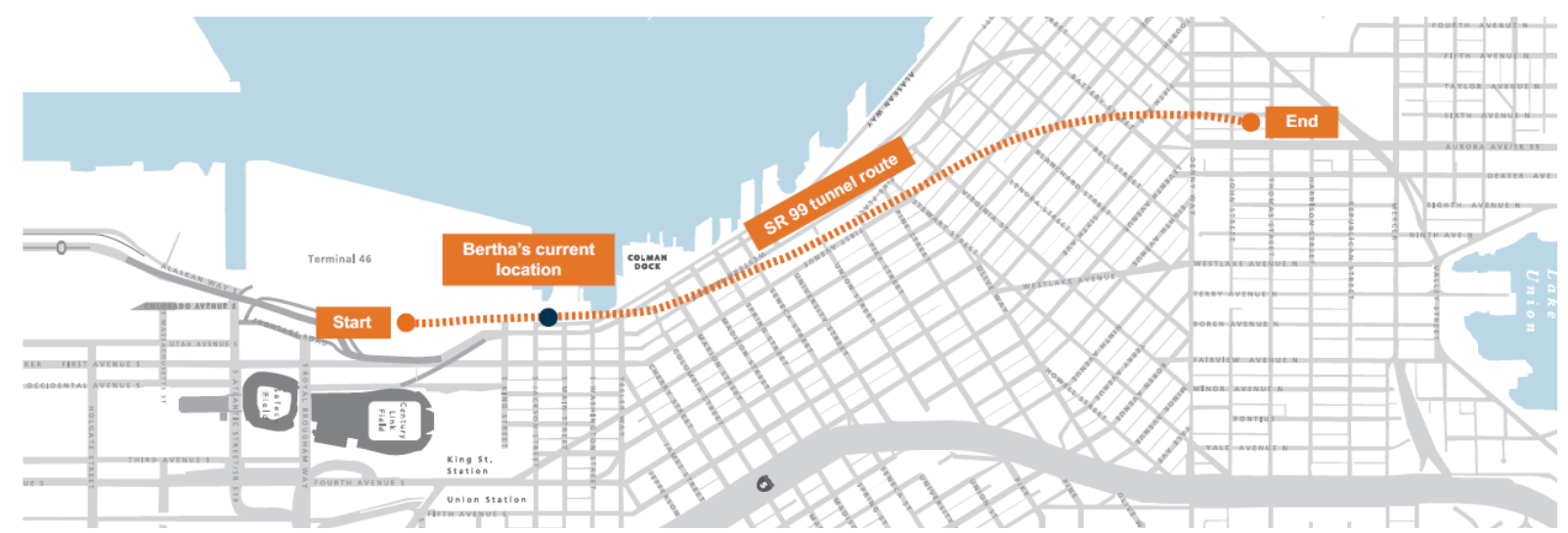

Figure 1 Planned tunnel route showing stalled location of tunnel boring machine 'Bertha' (courtesy WSDOT)

InSAR is a technology that allows for ongoing remote monitoring of surface deformation over wide spatial scales $10 \mathrm{~s}$ to $1,000 \mathrm{~s}$ of $\mathrm{km}^{2}$ (Massonnet et al. 1993). MDA has developed HDS-InSAR, which is an advanced InSAR solution designed to optimise the spatial resolution of mapped deformation by adaptively processing a broad class of scattering surfaces such as buildings, roadways and natural terrain.

Two RADARSAT-2 Spotlight mode ( $1 \mathrm{~m}$ resolution) image stacks from opposing look directions were acquired over the project site from 6 June 2012 to 2 February 2015 at 24-day intervals per stack (MacDonald, Dettwiler and Associates Ltd. 2014). This time period includes ten months prior to start of boring, the initial boring phase and the subsequent construction of the rescue shaft and associated dewatering.

HDS-InSAR was used to process the two RADARSAT-2 data stacks to derive multi-temporal surface deformation estimates along two satellite lines-of-sight (LOS). These estimates were then decomposed into separate time resolved maps of vertical and east-west deformation.

These maps were further decomposed into a set of temporal component maps that partition the observed deformation into the following components: (1) long-term linear deformation; (2) a component correlated with the onset of rescue shaft dewatering; and (3) thermally correlated deformation (in some cases corresponding to rigid structures dilating with ambient temperature). This decomposition has shown a wide scale $\left(\sim 1 \mathrm{~km}^{2}\right)$ area centred near the rescue shaft with significant deformation correlated with the onset of dewatering as well as significant smaller scale zones of linear deformation occurring throughout the monitoring period.

\section{$2 \quad$ Method}

\subsection{HDS-InSAR}

HDS-InSAR was used to process the RADARSAT-2 data stacks and generate multi-temporal surface deformation estimates along the LOS for each of the two data stacks. It uses adaptive multi-looking over local neighbourhoods with statistically similar amplitude distributions in order to supress noise over distributed targets while preserving point like targets (Parizzi \& Brcic 2011; Rabus et al. 2012). The adaptive filtering improves or maintains temporal coherence for multiple target classes such as buildings, roads and sparsely vegetated terrain during InSAR processing. This method is therefore well suited for urban infrastructure monitoring because it achieves higher effective spatial resolution compared to methods such as Differential InSAR and Persistent Scatterer InSAR in a mixed target environment. Figure 2 shows the HDS-InSAR processing steps. 


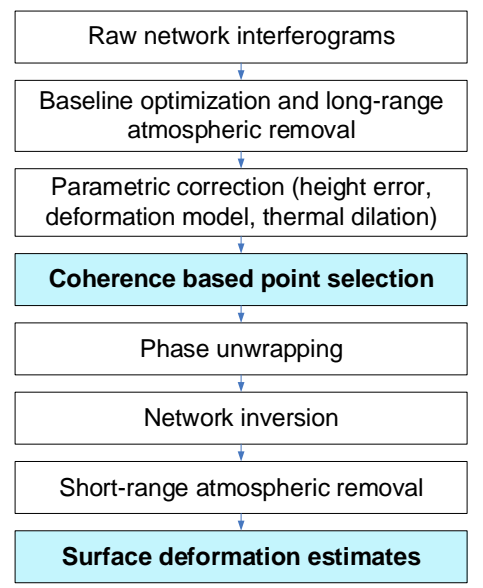

\section{Figure 2 HDS-InSAR processing steps}

\subsection{Temporal component modelling}

The surface deformation in the area surrounding the tunnel construction can in general be attributed to both pre-existing deformation conditions and those initiating during construction. It is therefore desirable to decompose the observed deformation into components correlated with these deformation drivers. To achieve this goal we implemented a simple linear parameterised temporal model fit to the estimated deformation by least squares (Rabus et al. 2012):

$$
d=\alpha f_{\text {linear }}+\beta f_{\text {delayed_linear }}+\gamma f_{\text {thermal }}+d_{\text {residual }}
$$

where:

$$
\begin{aligned}
& \mathrm{d}=\quad=\quad \text { deformation time history for single target (either vertical or east-west axis motion). } \\
& \mathrm{f}_{\text {linear }}=\quad \text { linear deformation basis function (unit slope) expected to correlate with pre-existing } \\
& \text { long-term deformation conditions. } \\
& \mathrm{f}_{\text {delayed_linear }}=\text { delayed linear deformation basis function (zero slope until onset of primary } \\
& \text { dewatering for rescue shaft, followed by unit slope) expected to correlate with } \\
& \text { deformation associated with dewatering. } \\
& \mathrm{f}_{\text {thermal }}=\quad \text { thermal deformation basis function (mean daily air temperature history) correlated } \\
& \text { with thermal expansion of structures as well as other seasonal effects such as near } \\
& \text { surface clay swelling. } \\
& \alpha, \beta, \gamma=\text { set of linear model coefficients derived for each target. } \\
& \mathrm{d}_{\text {residual }}=\text { residual (un-modelled) deformation. }
\end{aligned}
$$

The model basis functions are shown in Figure 3. This linear modelling generates a coefficient map for each of the three basis functions which provide a spatial view of the component contributions to the total deformation signal.

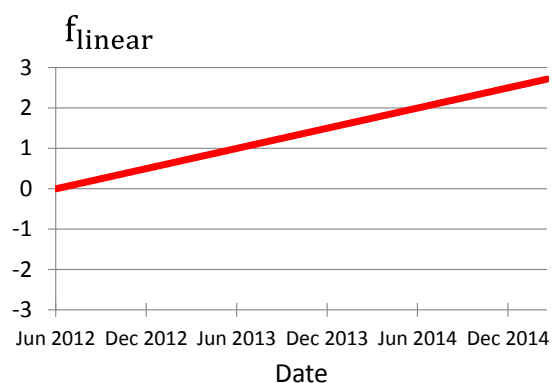

Date

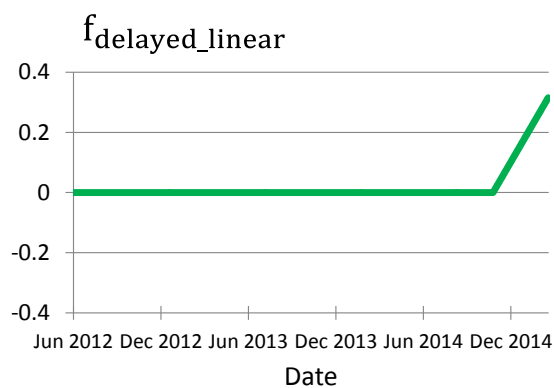

Date

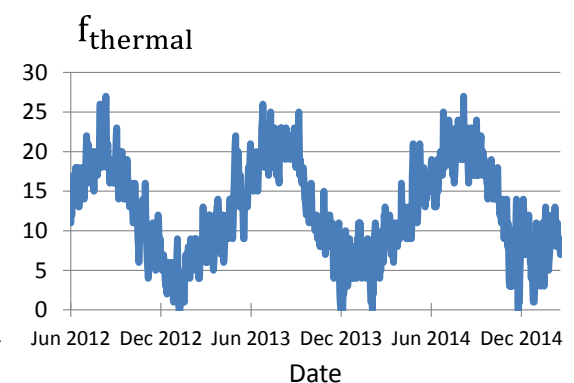

Date

Figure 3 Temporal component model basis functions 


\section{$2.32 D$ decomposition}

Two data stacks from opposing satellite pass directions were processed to derive a corresponding pair of LOS multi-temporal deformation estimates. Each LOS measurement corresponds to the projection of the true deformation vector along the sensor LOS. The two LOS vectors together form a basis over a 2D plane and it is therefore possible to compute orthogonal deformation components within this plane (SamieieEsfahany et al. 2009). Deformation components orthogonal to this plane are orthogonal to both stack LOS vectors and are therefore not observable by the paired measurements. As such, it is not possible to fully recover the 3D deformation vector given a pair of measurements. However, it is straightforward to solve for the specific solution with a minimum norm that corresponds to assigning a zero to the out-of-plane component, which is the approach taken in this study.

With the measurement geometry of opposing RADARSAT-2 pass directions the out-of-plane direction corresponds approximately to the north-south axis and therefore only vertical and east-west direction components are reported. It should be noted that since the out-of-plane component is not exactly aligned with the north-south axis, the estimated vertical and east-west components are biased by the out-of-plane component to a small degree.

A simulation is presented to demonstrate the utility of the 2D decomposition at estimating the vertical and east-west component of a circularly symmetric deformation field and to show the effect of measurement bias. Figure $4(\mathrm{a})$ shows a simulated circularly symmetric deformation field with $0.5^{*}(1+\cos (\mathrm{r}))$ radial profile and with horizontal deformation proportional to the vector spatial gradient of the vertical component. The LOS measurements corresponding to the geometry of the two image stacks used in this study are shown in Figure $4(\mathrm{~b})$. Note the east-west shift in the location of the peak LOS deformation between the two geometries.

A

$N$
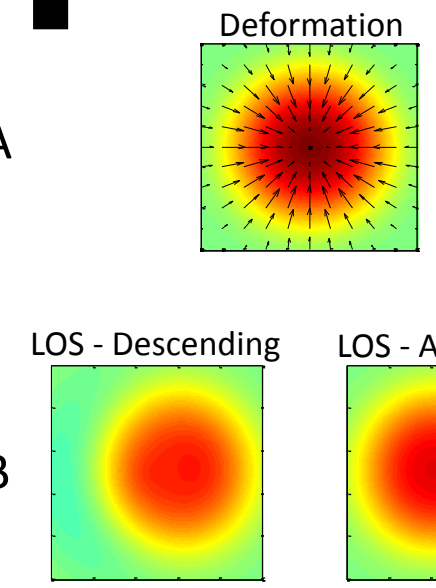

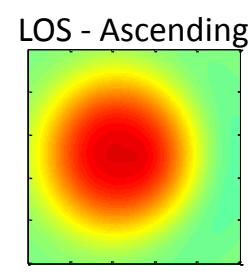

D

C
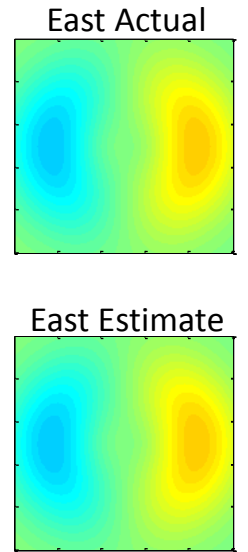

East Error

E

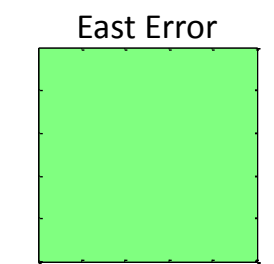

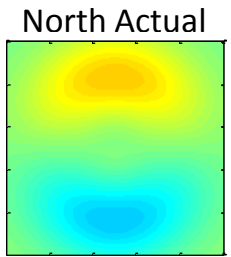

North Estimate

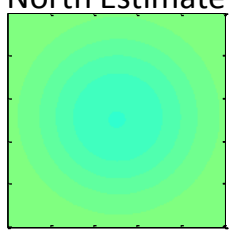

North Error

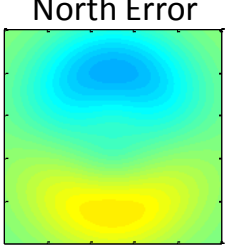

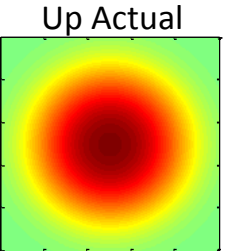

Up Estimate

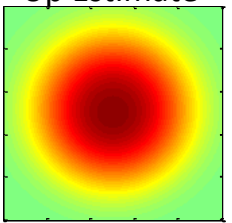

Up Error

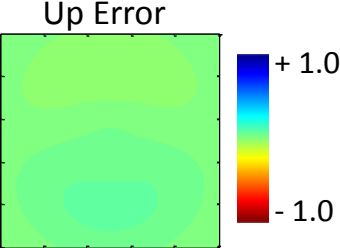

(c) MacDonald, Dettwiler and Associates Ltd. All Rights Reserved

Figure 4 (a) simulated deformation field. Colour depicts vertical deformation magnitude, arrows depict horizontal deformation magnitude and direction; (b) corresponding LOS components for the two image stack geometries; (c) (d) and (e) 2D decomposition simulation results showing actual deformation, estimated deformation and errors

Figures 4(c), (d) and (e) show the actual, estimated and bias components resulting from applying the 2D decomposition to the simulated LOS measurements. Examining the bias components shows that the west direction bias is negligible, the up component is small and coupled to the actual north component, and the 
north component bias comprises most of the actual north component. This simulation result validates the $2 \mathrm{D}$ decomposition in the case when the north component is of equal or lesser magnitude to the up component.

\subsection{Data description}

Two RADARSAT-2 Spotlight stacks from opposing satellite pass directions were acquired in order to allow for 2D decomposition of deformation estimates - see Table 1. Spotlight data have a resolution of $1.6 \mathrm{~m}$ in slant range and $0.8 \mathrm{~m}$ in azimuth (i.e. along satellite track) directions. These data were multi-looked during processing to produce images that have $\sim 3 \mathrm{~m}$ resolution in both ground range and azimuth directions. Figure 5 shows the ground footprints of the two stacks and the $8.7 \mathrm{~km}^{2}$ processed area of interest, which includes the southern $2 / 3$ of the planned tunnel and surrounding area.

Table 1 Summary of RADARSAT-2 data used for analysis

\begin{tabular}{ccc}
\hline & SLA9_Asc & SLA23_Des \\
\hline Pass direction & Ascending & Descending \\
Incidence angle & 37.0 & 46.7 \\
Number of scenes & 41 & 38 \\
Date range & $06 / 06 / 2012-15 / 02 / 2015$ & $06 / 06 / 2012-15 / 02 / 2015$ \\
\hline
\end{tabular}

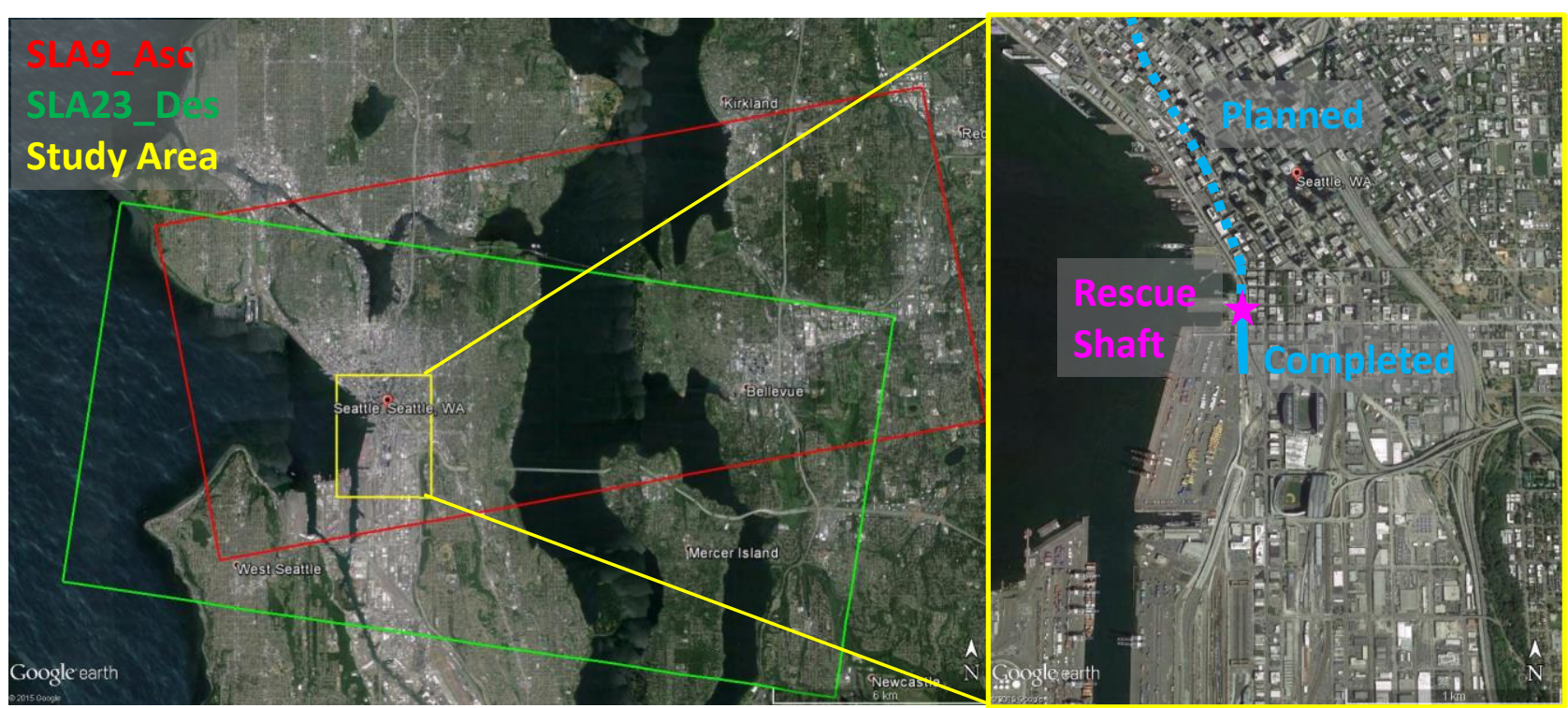

\section{Figure 5 RADARSAT-2 data footprint map showing per stack ground coverage and processed study area of interest}

Additional data required for InSAR processing include a digital elevation model (DEM) for topographic phase correction and ambient temperature data for estimation and removal of deformation due to thermal dilation of rigid structures. The DEM used is a 1/9 arc-second LiDAR derived DEM from the National Elevation Dataset courtesy of the US Geological Survey. Mean daily temperatures recorded at the Seattle-Tacoma International Airport were used as the thermal basis function for temporal component analysis. 


\section{$3 \quad$ Results}

\subsection{LOS deformation}

The ascending and descending RADARSAT-2 spotlight stacks were processed with HDS-InSAR to generate multi-temporal LOS deformation estimates. Figure 6 shows spatial maps of cumulative deformation for the 32-month data acquisition period. Note that positive values correspond to deformation toward the sensor and negative values correspond to deformation away from the sensor. Both view geometries show broad subsidence patterns with spatial extent $\sim 1 \mathrm{~km}^{2}$ in the immediate vicinity of the rescue shaft. The patterns show the ascending LOS subsidence to be greater in magnitude than that for the descending geometry and there is a several hundred meter east-west shift in the pattern centres between the two geometries. This shift is not unexpected given the difference in view geometries between the stacks.

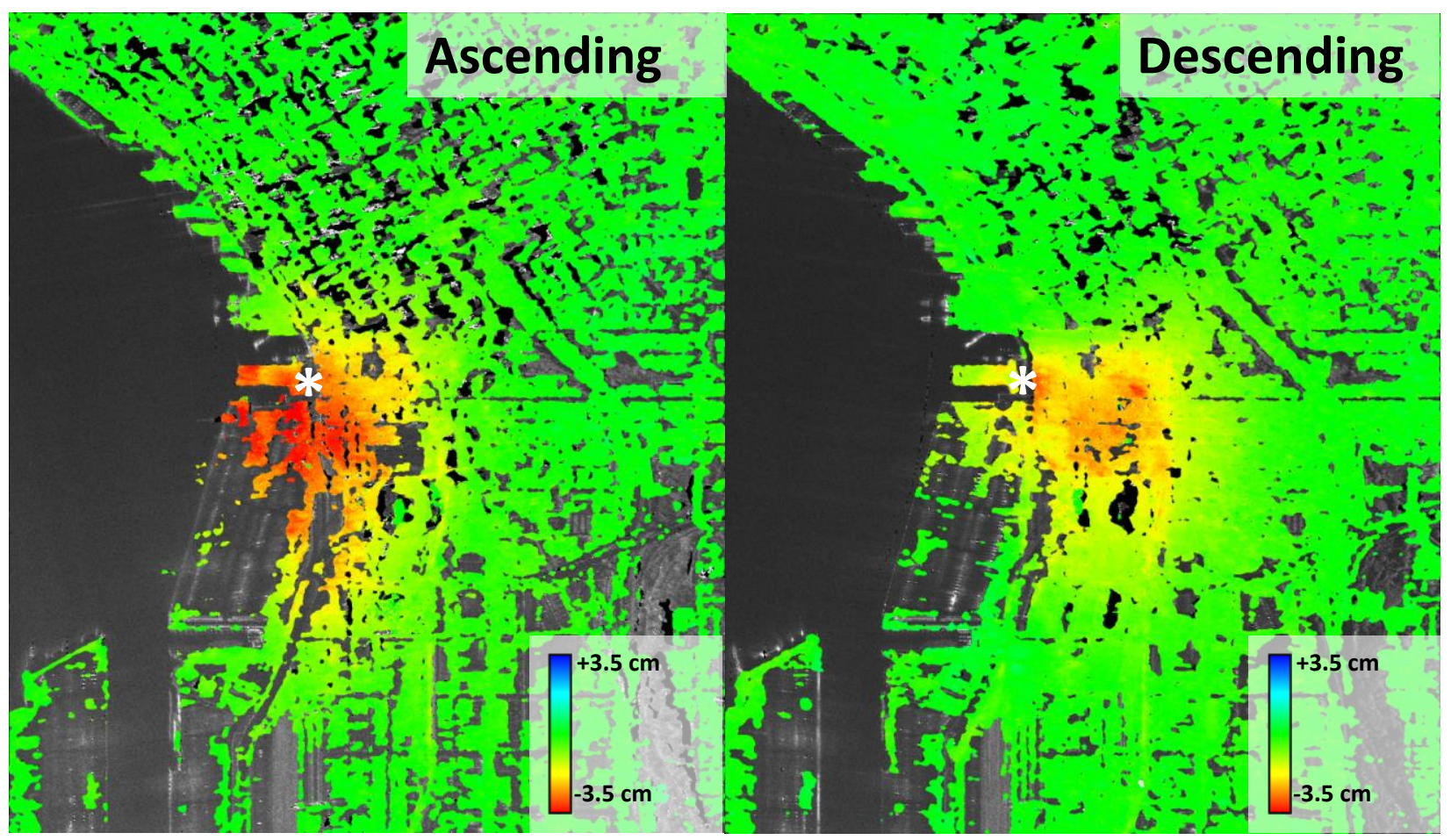

RADARSAT-2 Data and Products @ MacDonald, Dettwiler and Associates Ltd (2012-2015). All Rights Reserved. RADARSAT is an official mark of the Canadian Space Agency.

Figure 6 LOS cumulative deformation for the two data stacks (06/06/2012 - 15/02/2015). Location of rescue shaft is denoted with an asterisk

\subsection{D decomposed deformation}

The LOS deformation estimates were decomposed into vertical and east-west estimates as shown in Figure 7. Note that for the vertical case, positive values correspond to uplift and negative values correspond to subsidence. These indicate a $\sim 1 \mathrm{~km}^{2}$ roughly circular vertical subsidence pattern with a peak of $-3.5 \mathrm{~cm}$ over the monitoring period. For the east-west case, positive values correspond to eastward motion and negative values correspond to westward motion. The east-west component appears somewhat asymmetric with the eastward moving western side of the pattern having a greater north-south extent than the westward moving eastern side of the pattern. The peak east-west deformations have magnitudes of $\sim 1.5 \mathrm{~cm}$ over the measurement period. Both vertical and east-west patterns show fine-scale spatial structure corresponding to areas of increased strain. 


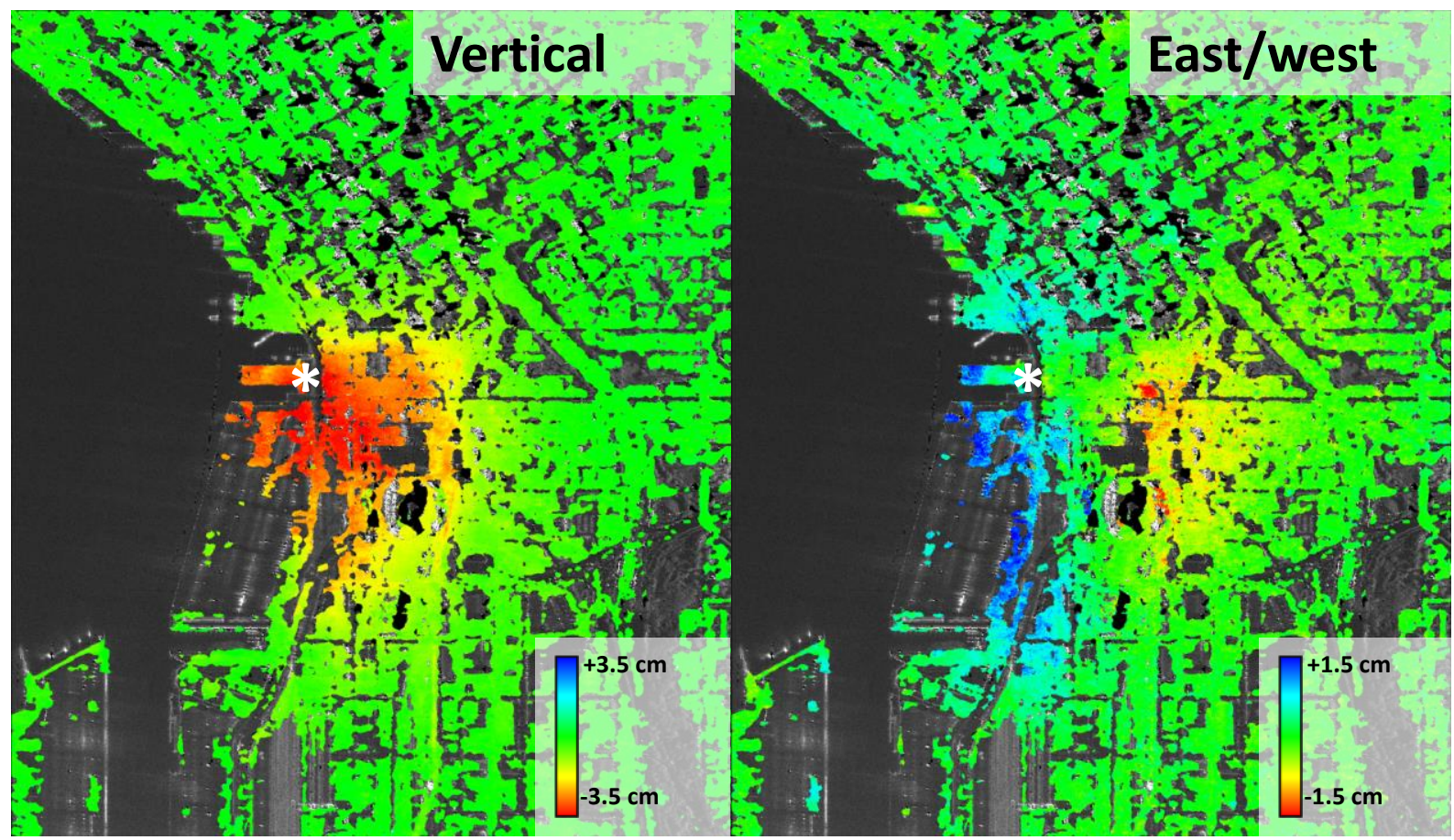

RADARSAT-2 Data and Products (C MacDonald, Dettwiler and Associates Ltd. (2012-2015). All Rights Reserved. RADARSAT is an official mark of the Canadian Space Agency.

\section{Figure 7 2D cumulative deformation (06/06/2012 - 15/02/2015). Location of rescue shaft is denoted with an asterisk}

\subsection{Temporal component modelling}

The linear three-component model was fit to the 2D deformation estimates to separate long term deformation and thermal expansion effects from the deformation correlated with onset of dewatering. Note that the delayed linear basis function was chosen to have the slope onset on $01 / 11 / 2014$, which corresponds approximately with onset of dewatering at the rescue shaft site. This date was chosen after examining the deformation results and noting a consistent change in slope near this date for both vertical and east-west components.

Figure 8 shows the three component coefficient maps and the model residual root-mean-square (RMS) map for the vertical deformation component. The delayed linear component is not centred at the rescue shaft but extends to the southeast. The peak rate is $-15 \mathrm{~cm} / \mathrm{y}$, which corresponds to $-4.4 \mathrm{~cm}$ deformation over the 3.5 months post-onset of dewatering. The linear component map shows many smaller areas with vertical deformation including the north/south oriented rail line running through the centre of the area of interest, along the existing viaduct and some buildings within the Pioneer Square district east of the rescue shaft. Deformation rates are generally less than $-0.5 \mathrm{~cm} / \mathrm{y}$. The vertical thermally correlated component is shown for completeness. This map shows many of the buildings positively correlated with temperature and this is likely due to thermal expansion. Other areas such as the docks on the waterfront are negatively correlated which may be due to seasonal affects related to the impact of water/humidity on the piled structures.

The RMS residual map shows that deviations from the model are spatially correlated with the delayed linear component. This is likely attributed to the fact that the dewatering correlated component is not entirely linear. The RMS values which include both noise and un-modelled deformation are low, as shown in the histograms in Figure 9. The mean residual RMS for the vertical model is $1.0 \mathrm{~mm}$. 


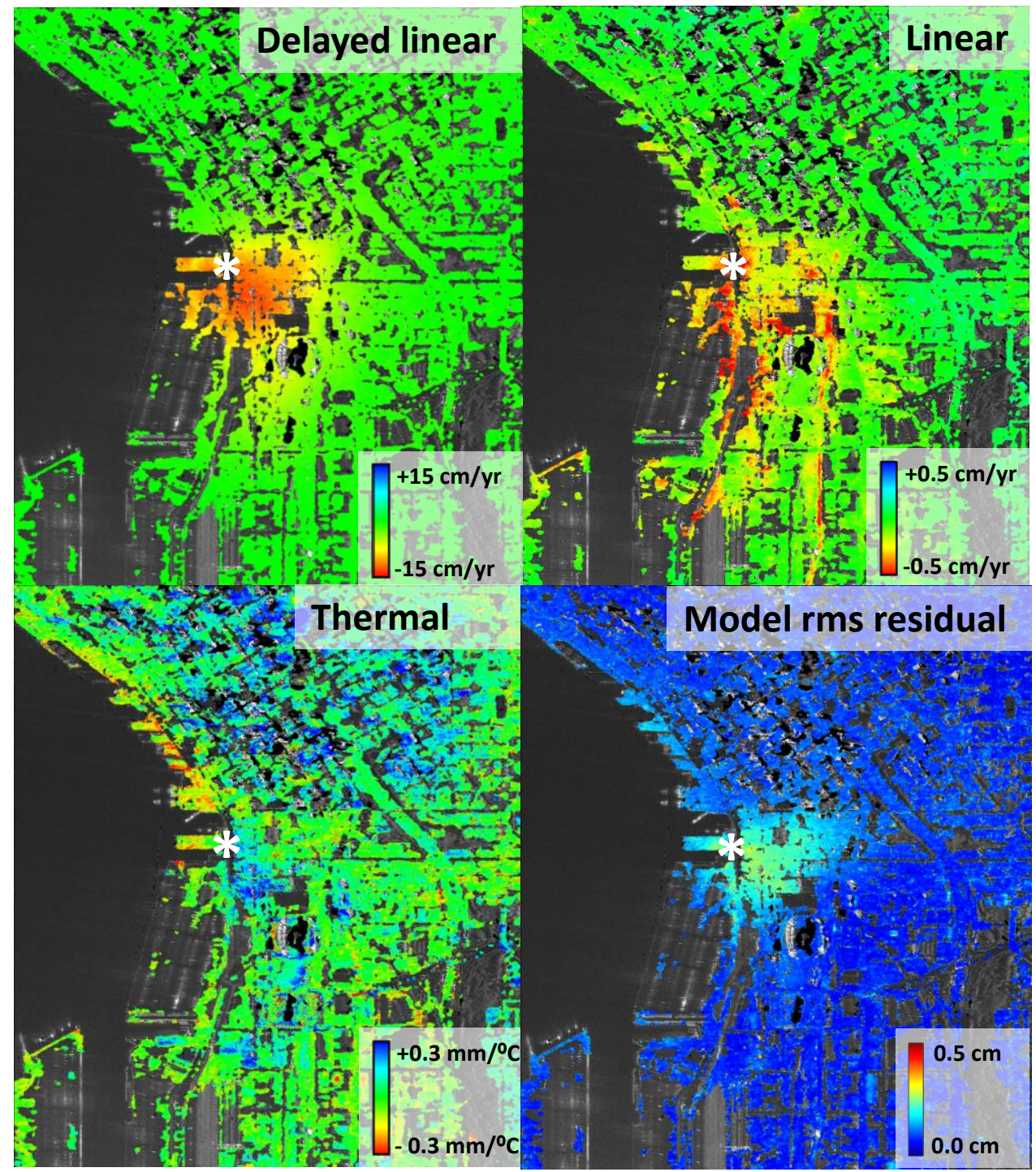

RADARSAT-2 Data and Products () MacDonald, Dettwiler and Associates Ltd. (2012-2015). All Rights Reserved. RADARSAT is an official mark of the Canadian Space Agency.

Figure 8 Temporal model coefficient maps for vertical deformation component. Location of rescue shaft is denoted with an asterisk

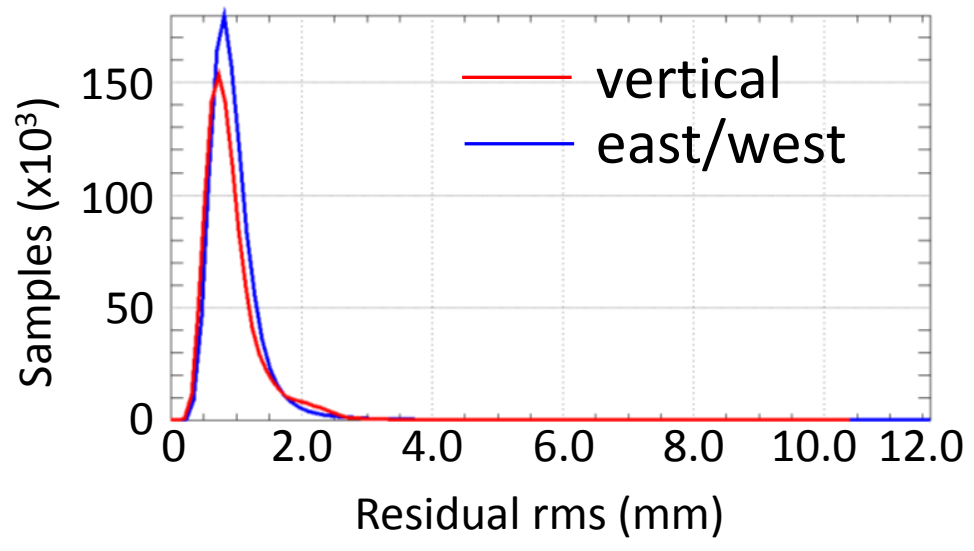

Figure 9 Distributions of model RMS residuals for both vertical and east-west components (vertical $=$ lower graph) 
Figure 10 shows the three-component coefficient maps and the model residual RMS map for the east-west deformation component. The delayed linear component shows the peak westward deformation occurring a few blocks east of the rescue shaft and the peak eastward deformation over the dock to the southwest. The peak rate magnitudes are $\sim 5 \mathrm{~cm} / \mathrm{y}$, which corresponds to $1.5 \mathrm{~cm}$ deformation over the 3.5 months post-onset of dewatering. The linear component map shows the long-term east-west deformation is predominately westward oriented with many areas exhibiting $\sim 0.2 \mathrm{~cm} / \mathrm{y}$ westward deformation. An area along the existing viaduct and a single building in the Pioneer Square district show westward deformation of $\sim 0.5 \mathrm{~cm} / \mathrm{y}$.

The east-west thermally correlated component is shown for completeness. This map shows many east-west oriented rigid structures thermally dilating including most notably the sports stadiums to the southeast of the rescue shaft.

The RMS residual map shows that deviations from the model are spatially correlated with the delayed linear component magnitude. As with the vertical component, this is likely attributed to the fact that the dewatering correlated component is not entirely linear. The RMS values are low as shown in the histograms in Figure 9. The mean residual RMS for the east-west model is $1.0 \mathrm{~mm}$.

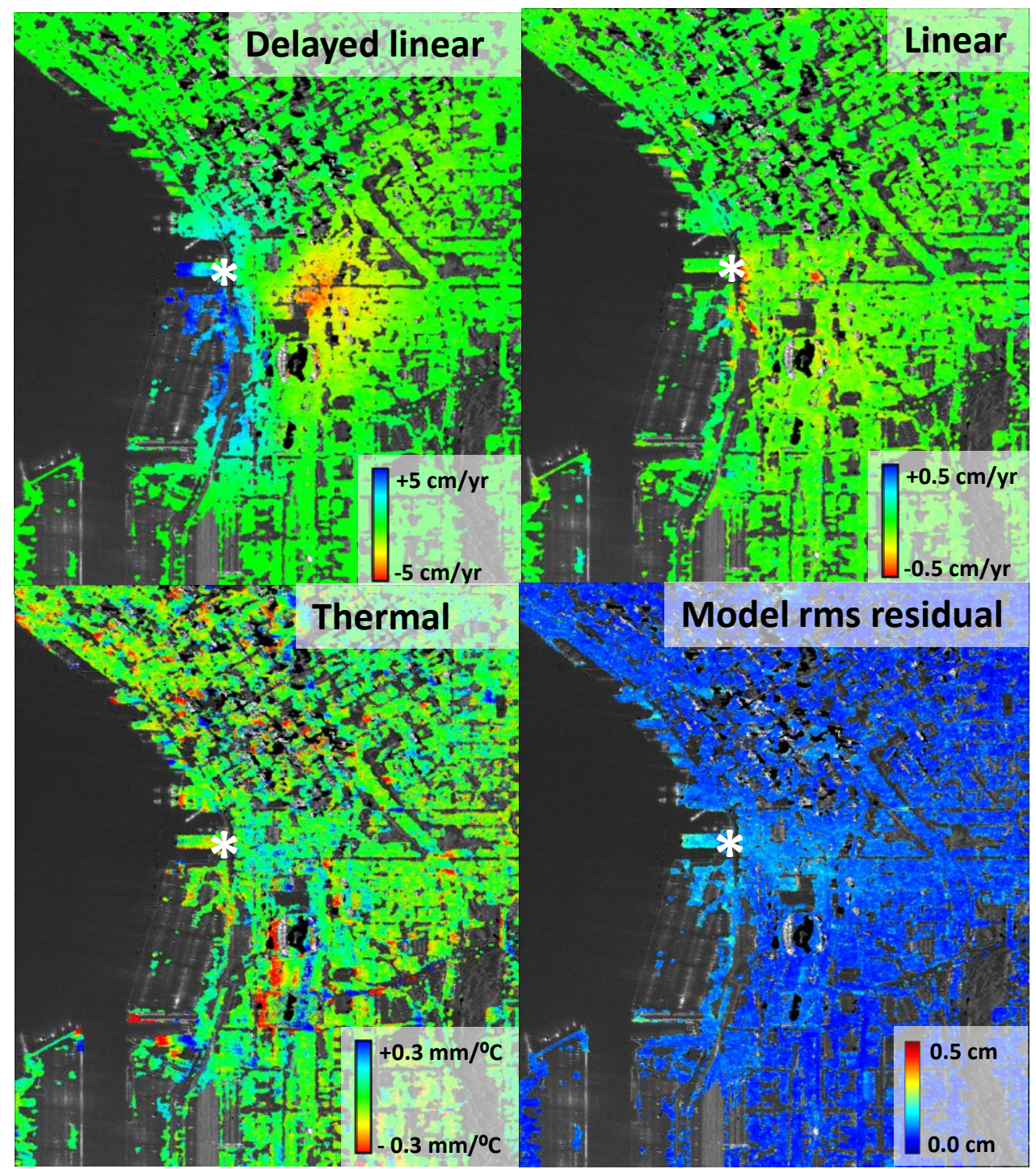

RADARSAT-2 Data and Products @ MacDonald, Dettwiler and Associates Ltd. (2012-2015). All Rights Reserved. RADARSAT is an official mark of the Canadian Space Agency.

\section{Figure 10 Temporal model coefficient maps for east-west deformation component. Location of rescue shaft is denoted with an asterisk}




\subsection{Deformation time series}

The 2D multi-temporal deformation estimates correspond to a vertical and an east-west deformation time series for every spatial target in the scene. Figure 11 shows some examples superimposed on the vertical linear component coefficient map for each point. Note that these time histories are shown with the thermal model component removed. Comparison of the model and deformation estimates shows that the chosen model fits the observed data well for the majority of points in the area of interest.
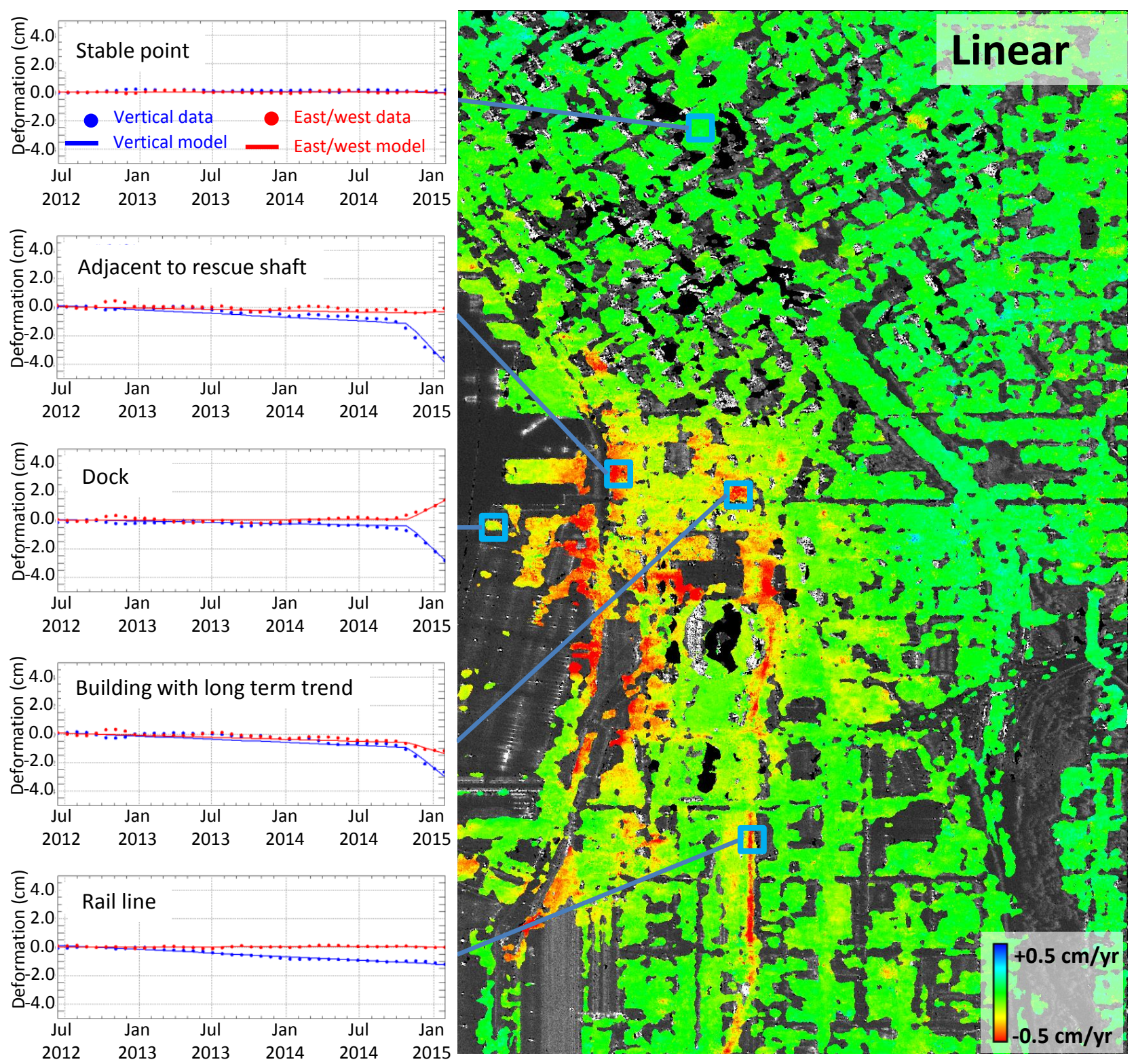

RADARSAT-2 Data and Products (c) MacDonald, Dettwiler and Associates Ltd. (2012-2015). All Rights Reserved. RADARSAT is an official mark of the Canadian Space Agency.

\section{Figure 11 2D deformation time series for select points. Plots show deformation estimates (points) superimposed with corresponding model with the thermal component removed from both}

\section{Conclusion}

This work presents an InSAR-based solution for monitoring tunnel construction in an urban setting. The solution was used to monitor the SR 99 Bored Tunnel Project. This project has included the excavation of a vertical rescue shaft to facilitate tunnel boring machine repair that has involved significant dewatering of the surrounding aquifer. 
The solution used RADARSAT-2 data and HDS-InSAR to provide LOS deformation estimates optimised for spatial resolution in a mixed target environment. Results from two view geometries were decomposed to recover both vertical and east-west multi-temporal deformation estimates. A linear three-component temporal model was fit to both the vertical and east-west deformation history of each monitored target.

The spatial density of coherent monitored targets was very high with almost continuous coverage in many areas. The 2D cumulative deformation maps for the 06/06/2012 - 15/02/2015 monitoring period showed significant deformation in both vertical and east-west directions in the area of interest primarily in the vicinity of the rescue shaft.

Residuals from the temporal component model were shown to have a mean RMS magnitude of $1.0 \mathrm{~mm}$ for both vertical and east-west components which showed that the model fit the data well for the majority of monitored targets. This was further supported by visual comparison of deformation time series to the corresponding temporal model curves.

Decomposed results showed significant vertical and east-west deformation correlated with the onset of rescue shaft dewatering with peak vertical values of $-4.4 \mathrm{~cm}$ vertical and $1.5 \mathrm{~cm}$ east-west magnitude over the (still continuing) 3.5-month dewatering period. This component has a spatial footprint of approximately $1 \mathrm{~km}^{2}$ in the vicinity of the rescue shaft. Linear component maps showed that there are smaller areas of significant long-term deformation occurring in the surrounding area with deformation rate magnitudes limited to $\sim 0.5 \mathrm{~cm} / \mathrm{y}$ per directional component. Notable examples include the existing viaduct, the rail line to the east of the rescue shaft, and some areas in the Pioneer Square district. The modelled thermally correlated components were shown to be consistent with a combination of structural thermal dilation effects and seasonal effects.

These results indicate that there is complex and significant deformation occurring in the vicinity of the rescue shaft site. This deformation includes both a long-term component and a more recent dewatering correlated component. These in turn include both vertical and east-west components and are likely to similarly include a north-south component, which was not estimated due to the sensor view constraints.

HDS-InSAR monitoring of the site with RADARSAT-2 Spotlight mode stacks therefore provided an effective solution for assessing the spatial and temporal deformation patterns in the area potentially affected by the tunnel construction activities.

\section{Acknowledgement}

We thank the Washington State Department of Transportation for their ongoing support for this study and the US Department of Transportation for funding this work.

\section{References}

MacDonald Dettwiler and Associates Ltd. 2014, RADARSAT-2 Product Description, MDA, Richmond, Canada.

Massonnet, D, Rossi, M, Carmona, C, Adragna, F, Peltzer, G, Feigl, K \& Rabaute, T 1993, 'The displacement field of the Landers earthquake mapped by radar interferometry', Nature, vol. 364, no. 6433, pp. 138-142.

Parizzi, A \& Brcic, R 2011, 'Adaptive InSAR stack multilooking exploiting amplitude statistics: a comparison between different techniques and practical results', IEEE Geoscience and Remote Sensing Letters, vol. 8, no. 3, pp. 441-445.

Rabus, B, Eppler, J, Sharma, J \& Busler, J 2012, 'Tunnel monitoring with an advanced InSAR technique', Proceedings of the SPIE, The international Society for Optical Engineering, Bellingham, vol. 8361, id. 83611F-83611F-10.

Samieie-Esfahany, S, Hanssen, R, Thienen-Visser, K \& Muntendam-Bos, A 2009, 'On the effect of horizontal deformation on InSAR subsidence estimates', in H Lacoste-Francis (ed.), Proceedings of the FRINGE Workshop on Advances in the Science and Applications of SAR Interferometry, ESA Communications, Noordwijk, $7 \mathrm{p}$.

Washington State Department of Transportation 2011, Alaskan Way viaduct replacement project: final environmental impact statement and section 4(f), WSDOT, Olympia, WA. 
